\title{
Punishment: effect on learning and resistance to extinction of discrete operant behavior'
}

\author{
Eileen B. Karsh 2 \\ DEPARTMENT OF BIOMEDICAL ENGINEERING, DREXEL INSTITUTE OF TECHNOLOGY
}

\begin{abstract}
Bar-pressing rate was reduced by shock but recovered when punishment stopped. Shock given during acquisition but not during extinction slowed the rate of extinction, compared to an unpunished control group. Extinction was more rapid for animals that had shock continued than for those that had shock removed during extinction.

\section{Problem}

Although resistance to experimental extinction is a traditional measure of response strength, relatively little work has been done to show the effect of both punishment and reward on this measure. Early studies by Skinner (1938) and Estes (1944) indicated that moderate punishment introduced during extinction did not hasten the extinction process. Logan (1960) showed that training with partial punishment and continuous reinforcement resulted in increased resistance to extinction. More recently Brown et al., (1964) found that under favorable circumstances, moderate punishment introduced during extinction could provide resistance to extinction.

The present experiment investigated long term effects of moderate punishment by examining behavior after cessation of punishment, both with reward present (recovery) and with reward removed (extinction). Specifically, it dealt with effects of shock on responding during acquisition and extinction of a bar-pressing response. Animals punished during training were given two extinction conditions: with or without punishment.

\section{Method}

Twenty-four male Sprague-Dawley rats, 150 days old, were maintained at $80 \%$ of normal body weight. Animals were fed once daily and were run $23 \mathrm{hr}$. later. The apparatus, described in detail in Karsh (1964), was a small box with a grid floor and a retractable bar enclosed in a larger, sound-resistant box.

A matched-impedance source with $150 \mathrm{~K}$ series resistor delivered a .15-sec. shock to the rat's feet through the bar and the grid floor immediately after the nineteenth bar press on shock trials.

The main response measure was pressing time (the time from the first to the nineteenth bar press). A reciprocal transformation was used in analyses and figures.

Animals were trained to press the bar 19 times in order to get a food pellet $(45 \mathrm{mg})$. Each rat was given magazine training and 100 reinforced bar presses, followed by 100 acquisition trials (which required 19 bar presses for one pellet).

In all conditions of the experiment, a trial consisted of the automatic presentation of the bar, 19 presses followed by the appropriate reinforcement contingency or the passage of $3 \mathrm{~min}$. with fewer than 19 bar presses followed by no reinforcement or punishment, and finally retraction of the bar. Ten trials were given daily; the interval between the end of one trial and the beginning of the next was $1 \mathrm{~min}$. During this interval, the house light was dimmed.

The rats were randomly assigned to three groups. The control group had 260 learning trials (with food and no shock) followed by 100 extinction trials (no food and no shock). There were two experimental groups, each at a different shock intensity: mild $(80 \mathrm{v})$ and moderate $(100 \mathrm{v})$. Each experimental group had four successive conditions: 100 learning trials (food alone), 50 shock trials (with food and shock), 60 recovery trials (food, no shock), and an additional 50 shock trials (with food). The second shock period was followed by extinction: no food and no shock for half the experimental animals and no foodbut the same shock as in training for the remaining experimental rats.

\section{Results and Discussion}

The animals increased their pressing rate in acquisition and reached a level of about 1.5 presses per sec. after 100 learning trials, but the control group increased to almost 2 presses per sec. after 200 trials, as shown in Fig. 1.

Both shock groups slowed their pressing rate during the first 50 shock trials, showing curves similar in shape to those in other experiments (Karsh, 1962,1964). After 50 shock trials, the three groups were reliably different (trials 131-150, shock intensity, linear: $\mathrm{F}=6.23, \mathrm{df}=1 / 21, \mathrm{P}<.025)$. When shock was stopped

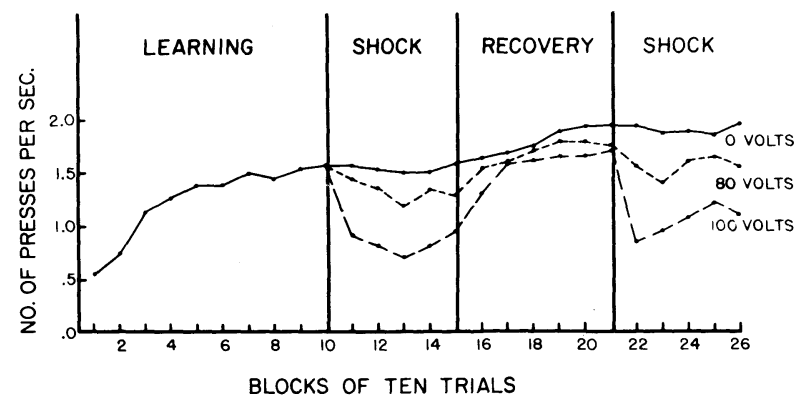

Fig. 1. Effect of presentation and removal of mild $(80 \mathrm{v})$ and moderate $(100 \mathrm{v})$ shock on rate of rats' bar pressing for food. The control group $(0 \mathrm{v})$ received no shock at any time and their performance is used as a baseline for comparison. 
and food was continued the two shock groups recovered fairly rapidly though they did not surpass the control. At the end of recovery the difference between the control and the experimental groups did not approach significance $(F<1)$.

In the second shock period, the two shock groups decreased their pressing rate at first, as before, and then increased somewhat. These curves, unlike those in the first shock period, are sigmoid in shape, as shown by a reliable cubic component. At the end of this shock period the three groups were again pressing at different rates (trials 241 260, shock intensity, linear: $\mathrm{F}=8.28, \mathrm{df}=1 / 21, \mathrm{P}<.01)$.

The extinction data are shown in Fig. 2. The first comparison is between the control group and the experimental rats that received no shock in extinction. These curves are labeled No Shock-No Shock and Shock-No Shock, respectively. The No Shock-No Shock curve is higher at the beginning of extinction and lower at the end. The group by trial block interaction was significant $(\mathrm{F}=8.17, \mathrm{df}=9 / 126, \mathrm{P}<.001)$, and it may be concluded that shock in acquisition increases resistance to extinction.

Another comparison is between the two groups of experimental rats that received either shock or no shock in extinction. The rats shocked during extinction

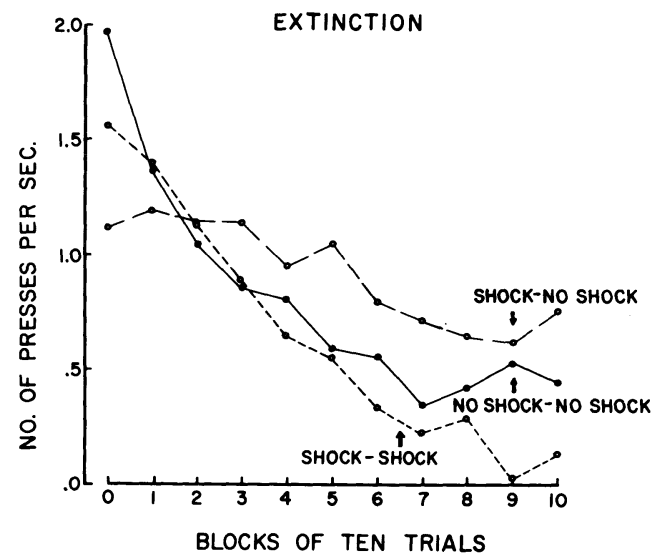

Fig. 2. Effect of removal of food and presence or absence of shock on rate of rats' bar pressing. Shock given during previous training with food is indicated before the hyphen; shock given in extinction is indicated after the hyphen.
(Shock-Shock in Fig. 2) showed more rapid extinction. This can be seen directly in Fig. 2 and was corroborated by statistical analysis $(F=3.61$, df $=9 / 108, P<.001)$.

The increased resistance to extinction shown by the group that had shock removed poses an interesting question. Does this resistance occur because cessation of shock produces a tendency for a depressed response to recover, and recovery counteracts to some extent the decrease in responding caused by removal of food? Another possibility is that punishment "fixates" responding. A rewarded and punished response can become more resistant to change because of stereotyped responses to shock conditioned during punishment training. Evidence of persistent punished responding has been found in previous experiments (Karsh, 1963). Further work is needed to assess the relative merits of recovery as opposed to response stereotypy in explaining the resistance to extinction of a previously punished response.

\section{References}

BROWN, J. S., MARTIN, R. C., \& MORROW, M. W. Self-punitive behavior in the rat: Facilitative effects of punishment on resistance to extinction. J.comp. physiol. Psychol., 1964, 57, 127-133.

ESTES, W. K. An experimental study of punishment. Psychol. Monogr., 1944, 57, No. 3.

KARSH, E. B. Effects of number of rewarded trials and intensity of punishment on running speed. J.comp. physiol. Psychol., 1962, 55, 44-51.

KARSH, E. B. Changes in intensity of punishment: Effect on running behavior of rats. Science, 1963, 140, 1084-1085.

KARSH, E. B. Punishment: Trial spacing and shock intensity as determinants of behavior in a discrete operant situation. J.comp. physiol.Psychol., 1964, in press.

LOGAN, F. A. Incentive. New Haven: Yale Univer. Press, 1960.

SKINNER, B. F. The behavior of organisms. New York: Appleton-Century, 1938.

\section{Notes}

1. This research was supported by grants MH 06951-01 and $\mathrm{MH}$ 08374-01 from the National Institute of Mental Health and by the Department of Psychology, Swarthmore College.

2. The author is indebted to L. Sharpe, C. Donal, J. Beardslee, and C. Lewis for assistance. 